\title{
Structural, electrical characterization of PANI/ZnO hybrid nanocomposites TFT for logic gates application
}

\author{
Naveen Kumar S K ${ }^{1 *}$, Vijaya Krishna ${ }^{1}$ and Omprakash $\mathrm{SS}^{1}$ \\ ${ }^{1}$ Dept. of Electronics, Mangalore University \\ Mangalore, Karnataka 574199, India. \\ Email: nave12@gmail.com
}

Thin Film Transistor (TFT) is the key elements for future day's electronic circuit, in which low cost, low temperature, vacuum free process and flexibility of the substrate are the need for the day. Organic polymer semiconductor's (OPS) have a unique feature, the suitable among OPS is Polyaniline (PANI), which is doped with Zinc Oxide ( $\mathrm{ZnO})$, and these Nanocomposites are coated on the Cellulose acetate substrate. Hybrid structure has been studied for its electrical characteristics, i.e. resistance of the film by I-V measurement unit and was found be $1 \mathrm{M} \Omega$. The thickness of the film was found to be $6000 \AA$. The surface roughness of PANI/ZnO are characterized by Atomic Force Microscope (AFM) was found to be $143 \AA$ The structure of TFT is achieved by coating electrodes on PANI/ZnO layer and the substrate. The input and output characteristics of TFT are carried out using I-V measurement system (Figure 1 and 2).

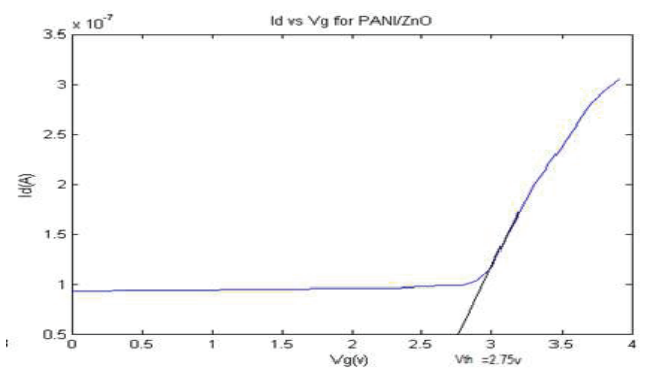

Figure 1: Input characteristics of Hybrid material

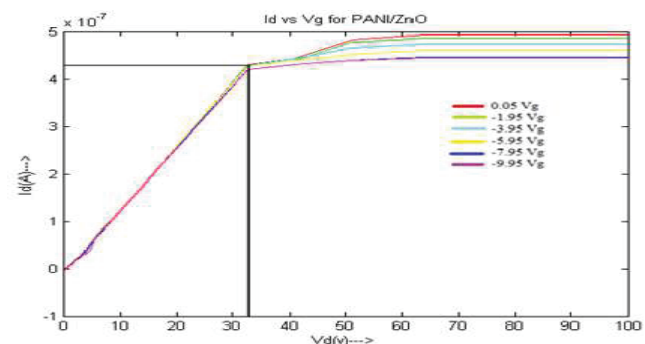

Figure 2: Output characteristics of Hybrid material
One of the basic building blocks of digital circuits is a NAND gate. Schematic of NAND gate [3] has been shown in Figure 3.

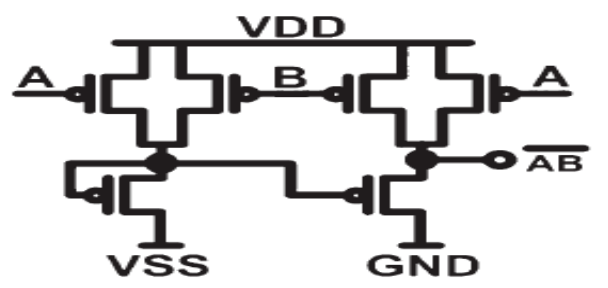

Figure 3: Schematic of NAND gate [3]

The NAND gate is designed, fabricated and tested for its output voltage. Table 1 sows the experimental NAND gate output.

Table 1: Experimental NAND gate output

\begin{tabular}{|c|c|c|}
\hline $\mathrm{A}$ & $\mathrm{B}$ & $(\mathrm{AB})^{\mathrm{I}}(\mathrm{v})$ \\
\hline 0 & 0 & 1.7 \\
\hline 0 & 1 & 1.68 \\
\hline 1 & 0 & 1.68 \\
\hline 1 & 1 & 1.34 \\
\hline
\end{tabular}

\section{References}

1. Wen-Chin Chiou, Jin-Lin Han, Sung-Nung Lee Synthesis and Studies of the Physical Properties of Polyaniline and Polyurethane-ModifiedEpoxy Composites. (2007)

2. Zhong Lin Wang Zinc oxide nanostructures: growth, properties and applications. (2004)R829R858

3. Tsung-Ching Huang, Member, IEEE, Kenjiro Fukuda, Chun-Ming Lo, Yung-Hui Yeh, Member, IEEE,Tsuyoshi Sekitani, Takao Someya, Member, IEEE, and Kwang-TingCheng, Fellow, IEEE Pseudo-CMOS: A Design Style for LowCost and Robust Flexible Electronics(2011) VOL. 58 